\title{
The Effects of Daily Melatonin Gavage on Reproductive Activity in the Male Syrian Hamsters
}

\author{
Geon Hyung Jeon', Hyeon Jeong Kim', Jinsoo Park², Sung-Ho Lee², Yong-Pil Cheon³, \\ and ${ }^{\dagger}$ Donchan $\mathrm{Choi}^{1}$ \\ ${ }^{1}$ Dept. of Life Science, College of Environmental Sciences, Yong-In University, Yongin 17092, Korea \\ ${ }^{2}$ Dept. of Biotechnology, Sangmyung University, Seoul 03016, Korea \\ ${ }^{3}$ Division of Developmental Biology and Physiology, Dept. of Biotechnology, Sungshin University, Seoul 02844, Korea
}

\section{Abstract}

The proper administration of melatonin has well been documented to induce testicular regression in seasonal breeding animals. The subcutaneous injections of melatonin in the afternoon, not in the morning, consistently occurred testicular involution in the male Syrian (golden) hamsters whose reproductive activity is regulated by the photoperiod. But the effects of daily melatonin via gavage have not been estimated. Golden hamsters housed in long photoperiod (LP) were divided into 5 groups: the control animals housed in LP or in short photoperiod (SP) and animals treated daily with low $(15 \mu \mathrm{g})$, middle $(150 \mu \mathrm{g})$, and high dosages $(1,500 \mu \mathrm{g})$ of pure melatonin by using gavage in the evening for 8 weeks. As results, LP control animals had large testes and SP controls displayed small and entirely regressed testes. The animals treated with various dosages of melatonin showed collectively degenerating effects on the weights of testes, epididymides, and seminal vesicles in the middle and high dosage groups, with the individual differences as well. The high dosages induced testicular regression in more proportion than the middle dosages did. The low dosage had large testes like the LP control animals. The small and inactive testes shown in some animals of both middle and high groups presented the complete regression as those of the animals maintained in SP. These results strongly suggest that the administrations of melatonin lead to testicular involution in the male golden hamsters when it is administered through gavage.

Keywords: Melatonin, Gavage, Reproductive activity, Male Syrian hamster

\section{INTRODUCTION}

Melatonin (N-acetyl-5-methoxytryptamine) discovered in 1950s has been investigated in the reproductive function of seasonal breeding animals for a long time (Lerner et al., 1958). It is produced mainly from the pineal gland, exhibiting a rhythmical pattern with elevated levels at night and with very low levels at day time (Rollag et al., 1980; Sugden 1989). The synthesis and secretion of melatonin are controlled by photoperiod.

Photoperiod is a major factor to control the reproductive functions of golden hamster, which is 
(2017R1D1A1B03028581).

\section{Authors' contributions}

Conceptualization: Lee SH, Cheon YP, Choi D.

Data curation: Jeon GH, Kim HJ, Park JS.

Formal analysis: Jeon $\mathrm{GH}$, Choi D.

Methodology: Jeon GH, Park JS, Choi D.

Software: Jeon GH, Choi D.

Validation: Lee $\mathrm{SH}$, Cheon YP, Choi D.

Investigation: Jeon GH, Kim HJ, Park JS.

Writing-original draft: Choi D.

Writing-review \& editing: Jeon GH, Lee SH, Cheon YP.

\section{Ethics approval}

This article requires IRB/IACUC approval because there are animal participants.

This study was approved by Yongin University Institutional Animal Care and Use Committee: YUIACUC-2019-02. a small mammal whose size is nearly intermediate between rat and mouse. Long photoperiod (LP, lighting of more than 12.5 hours in a day) like summer season maintains or promotes the reproductive activities (Gaston \& Menaker, 1967). While short photoperiod (SP, lighting less than 12.5 hours in a day) like winter season leads to entire regression of testis in 8 weeks of exposure (Stetson \& Watson-Whitmyre, 1984; Stetson \& Watson-Whitmyre, 1986; Choi \& Lee, 2012). Twelve and half hours of time in a day have been determined as the threshold of photoperiod, being a dichotomy boundary period of time that the reproductive activities are divided into active and inactive phases.

When the animals are exposed to a lighting at night, levels of melatonin are abruptly dropped into the levels of day time. Thus, the period of darkness in animals exposed to SP, in comparison to that in animals exposed to LP, lengthens relatively and elongates time period of synthesis and release of melatonin, leading to the reproductive degeneration (Stetson \& Watson-Whitmyre, 1984). In the end, it is thought that melatonin exerts its effects on the reproductive endocrine system in mammals.

Under the artificial light regimens, the same results have been occurred (Elliott, 1976). And it was revealed that each animal species had different lighting lengths of a day that promote the reproductive functions (Stetson \&Watson-Whitmyre, 1984; Stetson \&Watson-Whitmyre, 1986).

As SP presents longer period of darkness than LP, the period of elevated melatonin is relatively longer in SP, which is reflected by the length of melatonin secretion. Various modes of administration of melatonin into the animals, such as injections, implants, and infusions, have been applied whether they resulted in the testicular regression (Reiter 1980; Stetson \& Tay, 1983; Stetson et al., 1983; Stetson \& Watson-Whitmyre, 1984; Stetson \& Watson-Whitmyre, 1986; Maywood et al., 1991; Grosse et al., 1993; Choi, 2001; Hiebert et al., 2006; Choi, 2013a; Choi, 2013b). Some plants also contain melatonin called phytomelatonin, structurally an identical molecule (Dubbels et al., 1995; Hattori et al., 1995; Paredes et al., 2009). The findings raised an interest in investigating the potential effects of plant phytomelatonin on the reproductive activity of small animals, among the diverse capacities reported so far, particularly through the edible plant diet (Choi, 2013b; Bonomini et al., 2018; Jiki et al., 2018). The amounts of phytomelatonin that plants contain differ widely (Ramakrishna et al., 2012; Choi et al., 2014; Oladi et al., 2014; Meng et al., 2017; Arnao \& Hernández-Ruiz, 2018; Choi, 2019). The facts came across a possible action of phytomelatonin on the reproductive activity of male golden hamsters. The administration of an extract rich in phytometonin via gavage might induce testicular regression as the SP did. In order to measure a suitable dose of pure synthetic melatonin in inciting testicular involution, animals were subjected to pure melatonin by using gavage.

Therefore, the goal of the present work was to determine the dosage of melatonin administration through gavage in inducing testicular regression in the male golden hamsters.

\section{MATERIALS AND METHODS}

\section{Syrian hamsters}

Mature male golden hamsters (Mesocricetus auratus) were used in this experiment. They were maintained in animal breeding boxes within the animal breeding room. The animal breeding boxes were manufactured with wood and the period of time of lighting was controlled by the timer. The external lighting was blocked completely and ventilation was equipped with the small fans in one side. The animals were fed with standard laboratory mouse chow and tap water ad libitum and sanitary management was checked repeatedly. The animal breeding boxes set by LP 
(lights of 14 hours : darkness of 10 hours) or SP (lights of 10 hours : darkness of 14 hours) lighting condition were lined in a row. The lighting of animal breeding room outside the boxes was adjusted to identical to the SP condition and the point of middle time in day time of each photoperiod was synchronized in the ambient temperature of $22 \pm 1^{\circ} \mathrm{C}$. The condition of management of animals was approved by the Yongin University Institutional Animal Care and Use Committee (YUIACUC-2019-02).

\section{Photoperiod treatment and melatonin administrations}

The animals were divided into five groups: animals kept in LP lighting condition as LP control, animals treated with synthetic melatonin (Sigma-Aldrich, St. Louis, MO, USA ) of low (0.15 mg), middle $(1.50 \mathrm{mg})$, and high $(15.0 \mathrm{mg})$ dosages per $\mathrm{kg}$ of body weights, and animals transferred to SP lighting conditions as SP control. The administrations of synthetic melatonin were continued in daily gavage in a few hours before lights off for 8 weeks. Melatonin was primarily dissolved in $100 \%$ ethanol due to hydrophobic nature. And it was diluted with distilled water to make proper concentrations of melatonin in 10\% ethanol and applied instantly to the animals. The controls received daily $10 \%$ ethanol only as a vehicle.

\section{Determination of body and testes weights}

The body weights of the golden hamsters were weighed at two weeks intervals. And during the entire period of experimental time of 8 weeks, the unusual movement of hamsters was watched for minimal one hour immediately after melatonin intubation and at a certain hour of the day to inspect any abnormal aspects.

The volume of testes was measured by laparotomy at 0,4 , and 8 weeks. After the animals were anesthetized, the major axis and the minor axis of testis were rapidly measured by vernier calipers (Mitutoyo) after excising the skin overlying the scrotal sac and protruding the testes within the scrotal sac. The skin excised was sutured with autoclips (CLAY ADAMS ${ }^{\circledR}$ brand, MikRon ${ }^{\circledR}$ Precision, Biel, Switzerland). This procedure reduces the number of animals needed and thus is greatly economic without killing many animals (Watson-Whitmyre \& Stetson, 1985). At the end of the experiment, following the measurements of the testicular mass as performed above, the animals were sacrificed and the various internal organs, including testes, epididymides, and seminal vesicles as reproductive organs, were isolated and directly weighed. The testes and epididymides were kept in formalin until use for histological examination.

The testicular measures were converted into testicular mass by calculating the major axis and the minor axis via a convenient formula developed previously (Watson-Whitmyre \& Stetson, 1985). The calculated masses of testis were compared, plotted, and analyzed with the testicular weights straightly weighed.

\section{Histological examination}

Histological examination was performed by using paraffin tissue section. The testicular tissues were fixed in formalin for the time. Fixed tissues were dehydrated in graded concentrations of ethanol (70\%, 80\%, 90\%, 95\%, and 100\%) for 1.5 hours with gentle shaking and soaked in absolute ethanol overnight. The tissues were immersed in xylene three times for 30 minutes and in paraffin at $56^{\circ} \mathrm{C}$ three times for 30 minutes. They were embedded in paraffin and sectioned at $5 \mu \mathrm{m}$. The slices were mounted on microscope slide glasses and the slides were stained with hematoxylin (Sigma-Aldrich) and eosin (Sigma-Aldrich) for 5 minutes, respectively. They were dried entirely to evaporate and treated with canada balsam (Sigma-Aldrich) for permanent specimen, and observed under microscope. 


\section{Statistical analysis}

Data were expressed as mean \pm SD. Statistical analysis was performed using analysis of variance and student's t-test. Differences were considered to be significant at $p<0.05$.

\section{RESULTS}

\section{Changes of body weight}

The body weights of golden hamsters were not changed abruptly, matching with usual growth paradigm (Table 1). No significant differences of body weights were observed among any experimental groups at the end of this experiment. As the time went by, there was no any particular aberrant action in animals treated with melatonin by the daily gavage. Thus, the melatonin administration by gavage was concluded not to affect body weights and not to cause any atypical behavior.

\section{Changes of testes}

\section{1) Final testicular weights at the end of experiment}

At the end of experiment the actual weights of testes were indeed weighed. The weights of paired testes are shown in Fig. 1. The testes of animals housed in LP were large and heavy but those in SP very small and light. In the animals treated with pure melatonin, there was a significant difference. Low dose animals had large testes as the LP control animals, but they showed significant differences of testes weights to the middle and high dosage animals $(p<0.05)$, whose testicular weights were also significantly different to the SP control animals who showed all the complete regression $(p<0.05)$. And the middle and high dose groups showed uneven results, which means some differences of individual responses to melatonin on testicular activity.

\section{2) Average changes of testicular masses with time}

The testicular masses were calculated by the major axis and the minor axis and were used instead of the real testicular weights (Watson-Whitmyre \& Stetson, 1985). The average changes of testicular mass of golden hamsters who received various concentrations of melatonin via gavage are shown in Fig. 2. At the beginning of this experiment all animals had the large testes. they also showed relatively large testes at the 4th week, although the testes were a little diminished, still

Table 1. Changes of body weights of hamsters treated with melatonin

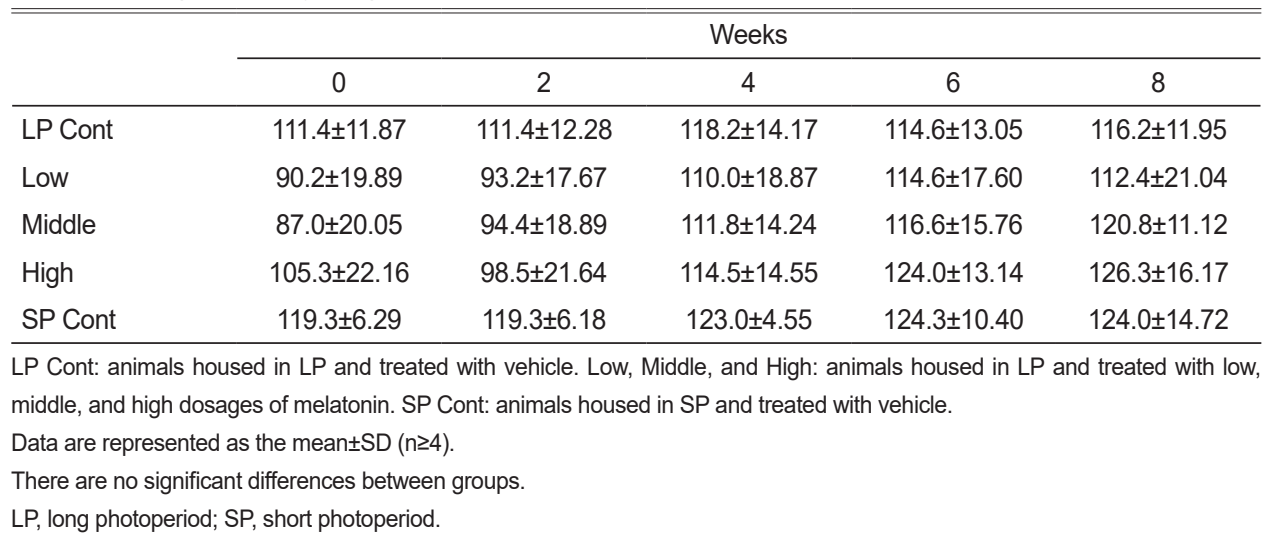




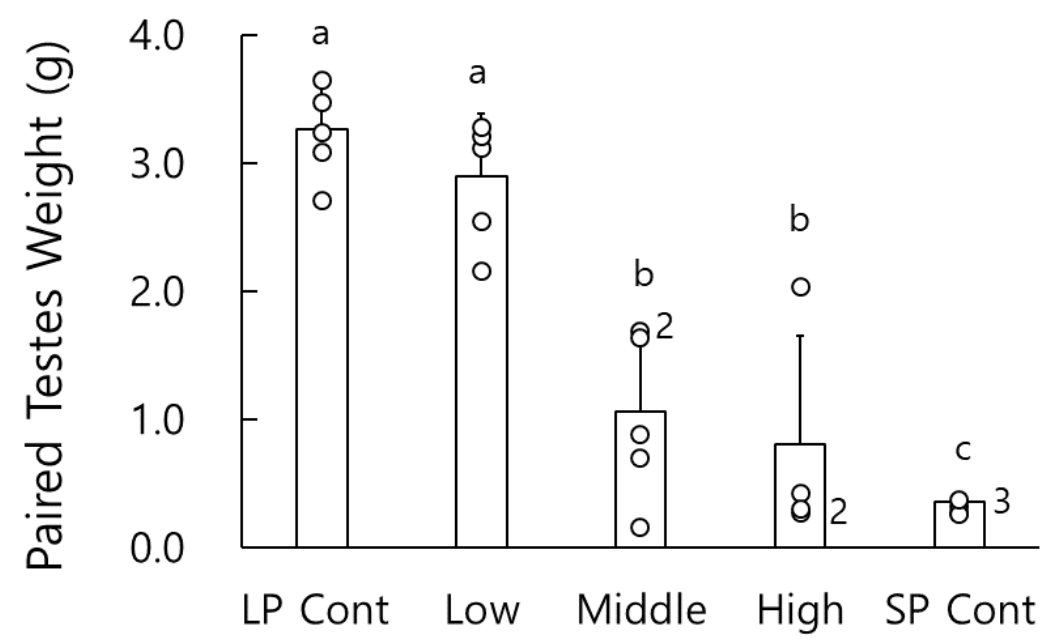

Fig. 1. Changes of actual testicular weights of golden hamster at the end of experiment. The testicular weights of individual animal are represented by tiny rings. The digits at the right side of the bars indicate the number of the open close circles. Note that some animals in middle and high group showed completely regressed testes. LP Cont: animals housed in long photoperiod (LP) and treated with vehicle. Low, Middle, and High: animals housed in LP and treated with low, middle, and high dosages of melatonin. SP Cont: animals housed in short photoperiod (SP) and treated with vehicle. Different letters indicate statistical significance $(p<0.05)$.

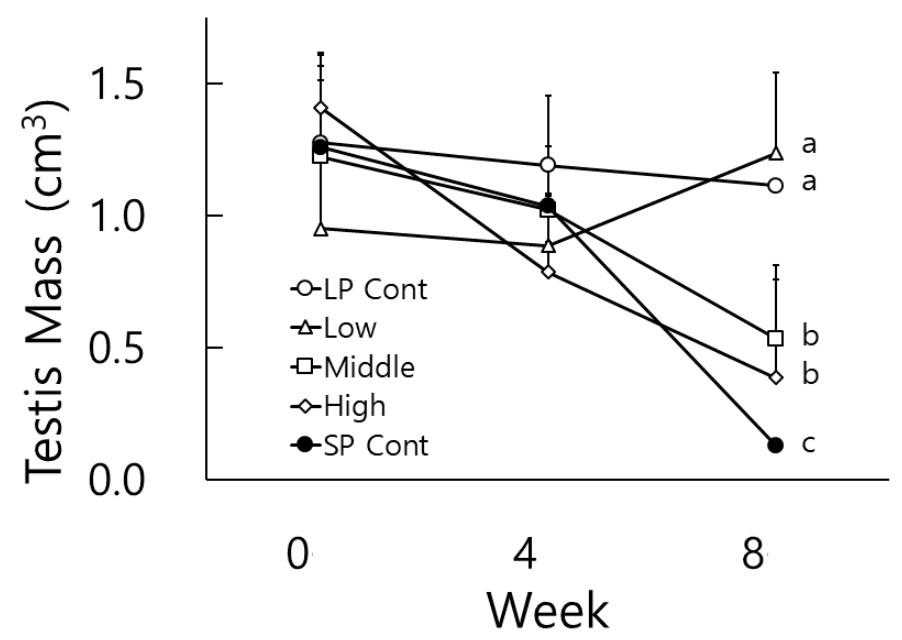

Fig. 2. Changes of testicular mass of golden hamster. The testicular masses of golden hamsters were gauged at 4 weeks intervals. LP Cont: animals housed in long photoperiod (LP) and treated with vehicle. Low, Middle, and High: animals housed in LP and treated with low, middle, and high dosages of melatonin. SP Cont: animals housed in short photoperiod (SP) and treated with vehicle. The digits at the right side of the bars indicate the number of the open close circles. Different letters indicate statistical significance $(p<0.05)$.

maintaining the active spermatogenesis. At the 8th week the average masses of testes of LP control animals and low dose animals were significantly different from those of middle and high dosage groups $(p<0.05)$. And similar to the outcome of the paired testes weights mentioned above, the middle and high dosage groups also displayed significant differences of testicular masses to the SP control animals $(p<0.05)$.

\section{3) Individual changes of testicular mass with time}

The testicular masses of individual animals in each group are also indicated in Fig. 3. There were 


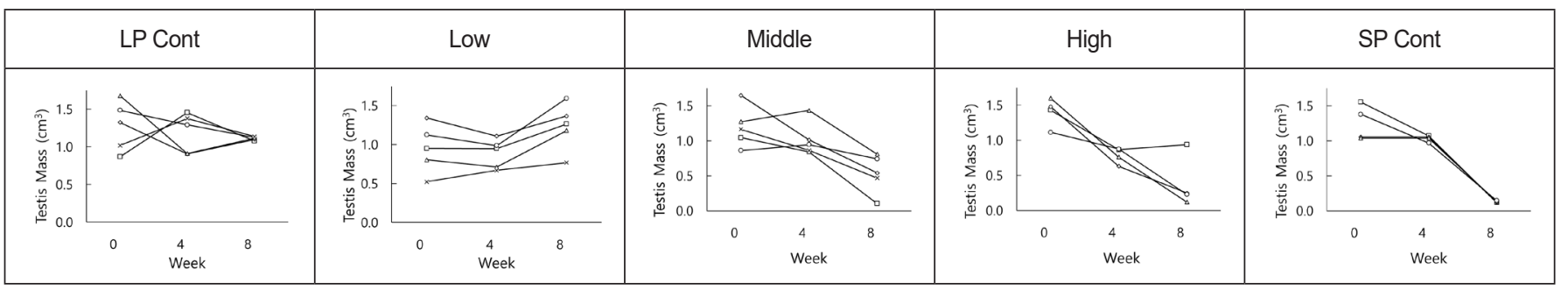

Fig. 3. Changes of testicular mass in individual animals. LP Cont: animals housed in long photoperiod (LP) and treated with vehicle. Low, Middle, and High: animals housed in LP and treated with low, middle, and high dosages of melatonin. SP Cont: animals housed in short photoperiod (SP) and treated with vehicle.

apparent individual differences at the end of the present experiment in groups treated with the synthetic melatonin. Throughout this experiment each animal in LP control group had large testes (Fig. 3, LP Cont) and that in SP group displayed very small testes (Fig. 3, SP Cont), indicating reproductive active and inactive testes, respectively. All animals in low dose groups showed definitely large testis (Fig. 3, Low). But some animals in both middle and high dose groups showed definitely complete regression of the testes (Fig. 3, Middle \& High). If the presence and absence of spermatozoa in the testis is regarded by histological examination as a criterion to distinguish gonadal regression, 1 animal out of 5 animals in middle group and 3 animals out of 4 animals in high group presented entire involution of testis. A few animals in middle dosage group had testicular masses to be less than the testicular masses of the animals in LP control group, which was speculated as a progression of testicular regression.

Although the numbers of animals of each group were not enough to assert clear-cut effect of synthetic melatonin, these results that occurred on the gonadal regression were evident.

\section{Testicular masses calculated versus testicular weights weighed}

The testicular masses calculated from the axes and the testes actually weighed at the end of experiment were compared to inspect the correlation between mass and weight. The results are plotted in Fig. 4 . As the correlation coefficient is near to $1\left(\mathrm{R}^{2}=0.93\right)$, it could be estimated that the

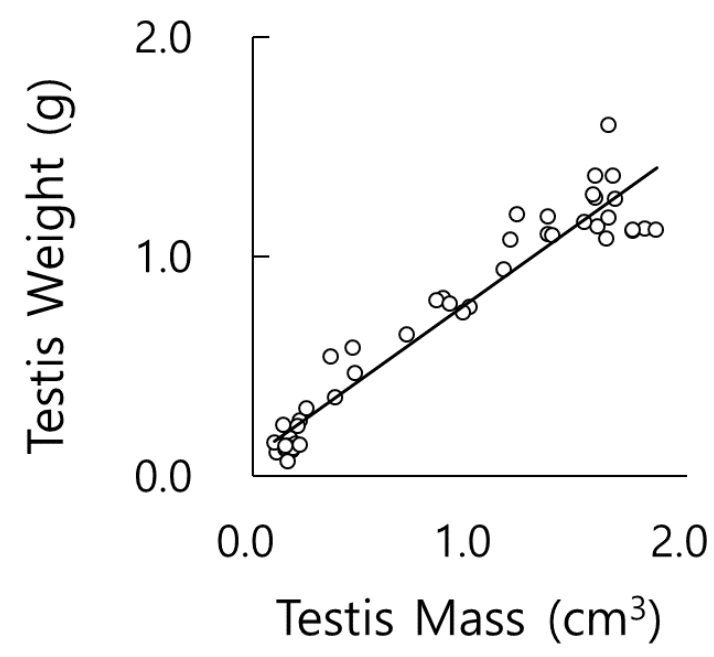

Fig. 4. A correlation between the testicular masses and the real testicular weights. Abscissa shows the values calculated from the major and the minor measures and ordinate the real values weighed. As the correlation coefficient is near to 1 , it could be estimated that the masses converted from the measures replace the weights actually weighed $(n=46)$. 
values converted from the mass exhibited the values weighed actually as reported previously (Lee et al., 2013). The outcome implies that the values measured could completely replace in a close parallel the values weighed.

\section{Weights of epididymides and seminal vesicles at the end of experiment}

Fig. 5 shows the effects of melatonin ingestion on the average weights of epididymides and seminal vesicles. Unlikely the results of testes, the weights of the epididymis and the seminal vesicle in the LP control animals were significantly different to those in low dosage animals $(p<0.05)$. And animals treated with low dose melatonin showed significant difference $(p<0.05)$ in weights of both epididymides and seminal vesicles in comparison to middle, high dosage groups, and SP controls. The reproductive accessory organs were apparently reduced in all the animals who had the regressed testes by ingestion of melatonin as the animals housed in SP control.
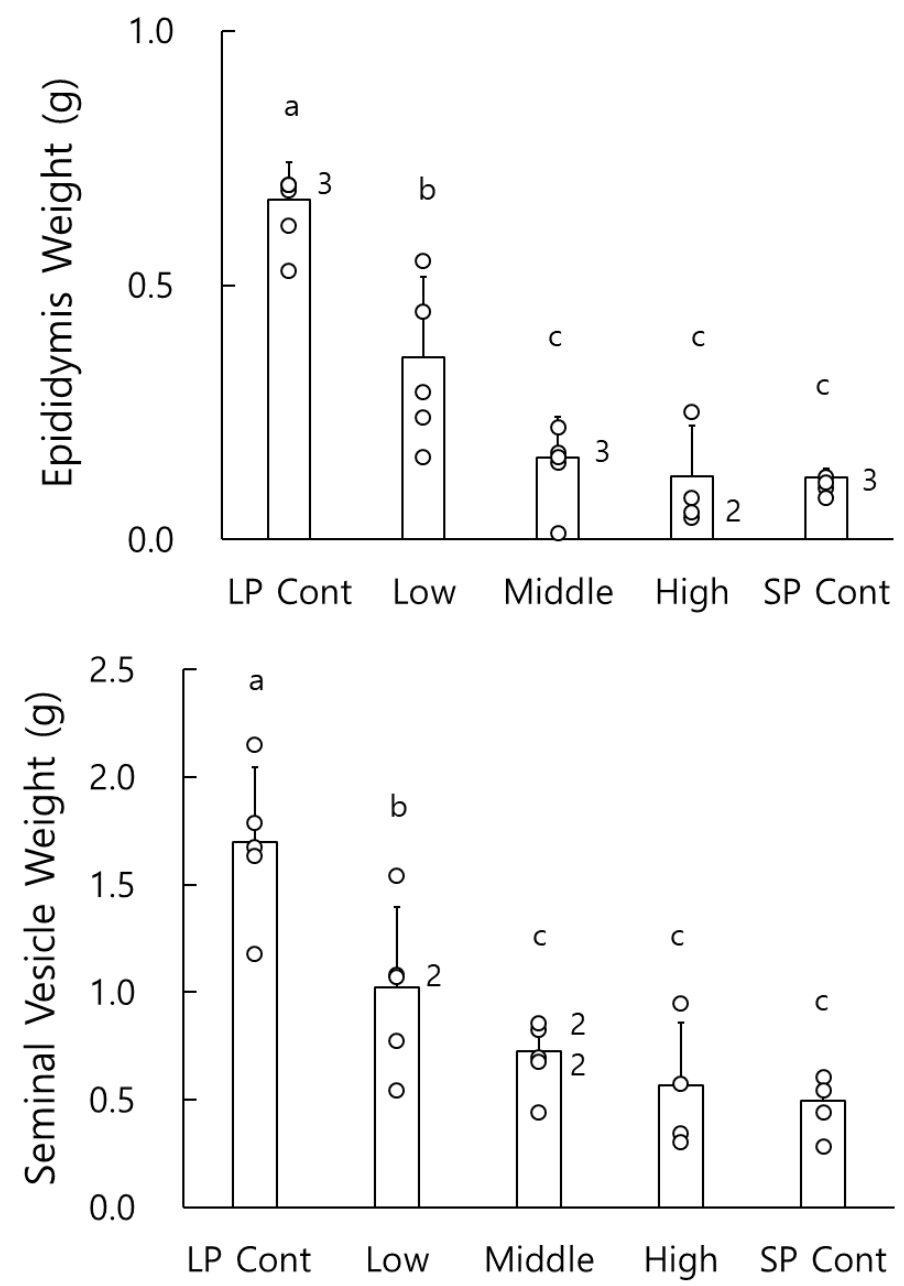

Fig. 5. Changes of weights of epididymis and seminal vesicles of golden hamsters at the end of experiment. The weights of individual animal are represented by tiny rings. The digits at the right side of the bars indicate the number of the open close circles. Note that some animals in middle and high group showed completely regressed epididymis or seminal vesicles. LP Cont: animals housed in long photoperiod (LP) and treated with vehicle. Low, Middle, and High: animals housed in LP and treated with low, middle, and high dosages of melatonin. SP Cont: animals housed in short photoperiod (SP) and treated with vehicle. Different letters indicate statistical significance $(p<0.05)$. 


\section{Weights of various organs at the end of experiment}

In order to survey any alterations of internal organs, various organs were isolated and weighed at the end of experiment (Table 2). The weights of the other organs that were unrelated to the reproductive activities were not noticeably altered by the melatonin ingestion.

\section{Histological examination of testes and epididymis}

In this experiment the testes could be classified into three categories, which are active, inactive, and regression-undergoing testes (Fig. 6). The active testes were found in LP control and low dose melatonin-treated animals and marked as Non-Reg, meaning non-regression (LP, Non-Reg). The regression-undergoing testes were observed in some animals in middle and high dosage groups and marked as partial reg (Partial Reg). The inactive testes were shown in some animals of middle and high dosage groups and all animals of SP control group, and marked as comp reg, meaning complete regression (SP, Comp Reg).

The active testis showed all kinds of germ cells, including spermatogonia, spermatocytes,

Table 2. Changes in weights of various organs

\begin{tabular}{cccccc}
\hline \hline & LP Cont & Low & Middle & High & SP Cont \\
\hline Heart $(\mathrm{g})$ & $0.6 \pm 0.14$ & $0.5 \pm 0.16$ & $0.4 \pm 0.12$ & $0.4 \pm 0.07$ & $0.6 \pm 0.10$ \\
Lung $(\mathrm{g})$ & $0.9 \pm 0.20$ & $0.7 \pm 0.07$ & $0.9 \pm 0.27$ & $0.8 \pm 0.11$ & $1.0 \pm 0.23$ \\
Liver $(\mathrm{g})$ & $4.6 \pm 0.46$ & $2.5 \pm 0.59$ & $3.1 \pm 1.09$ & $2.4 \pm 0.46$ & $4.6 \pm 0.58$ \\
Kidney $(\mathrm{g})$ & $1.0 \pm 0.13$ & $0.8 \pm 0.12$ & $0.8 \pm 0.09$ & $0.8 \pm 0.04$ & $1.0 \pm 0.13$ \\
Spleen $(\mathrm{g})$ & $0.17 \pm 0.035$ & $0.11 \pm 0.013$ & $0.16 \pm 0.057$ & $0.14 \pm 0.026$ & $0.14 \pm 0.041$ \\
\hline
\end{tabular}

LP Cont: animals housed in LP and treated with vehicle. Low, Middle, and High: animals housed in LP and treated with low, middle, and high dosages of melatonin. SP Cont: animals housed in SP and treated with vehicle.

Data are represented as the mean $\pm S D(n \geq 4)$.

There are no significant differences between groups in each organ.

LP, long photoperiod; SP, short photoperiod.

\section{LP, Non-Reg}
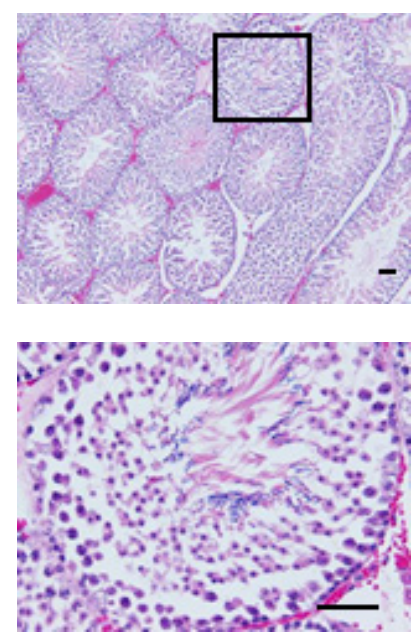

Partial Reg
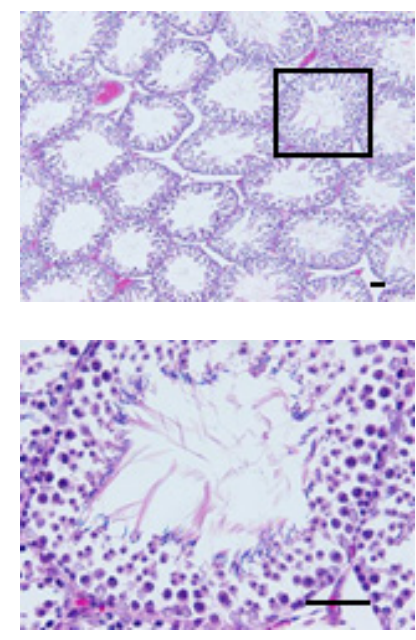

SP, Comp Reg
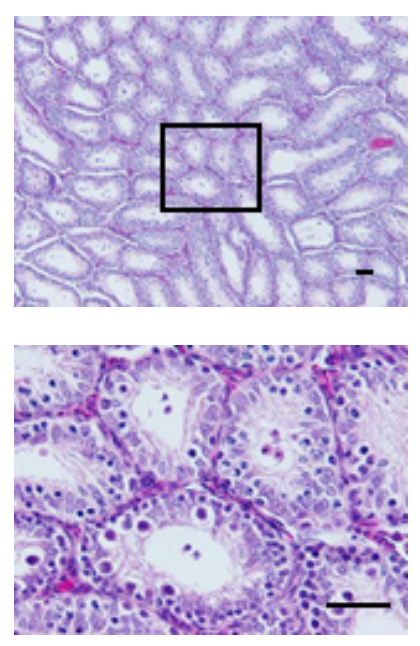

Fig. 6. Representative histological view of testis. LP, Non-Reg: testis of animals in long photoperiod (LP) control and animals who had non-regressed testis. Partial Reg: testis of animals who showed partial regression. SP, Comp Reg: testis of animals in short photoperiod (SP) control and animals who had completely regressed testis. The rectangles in upper row are amplified in lower row. Bar=50 $\mu \mathrm{m}$. 
spermatids, and spermatozoa (LP, Non-Reg). These results were evident in the thickened diameter of the seminiferous tubules and abundance of germ cells in the epithelium of the tubules of LP animals compared to those of SP animals. The average diameter of the tubules of active testis was near $280 \mu \mathrm{m}$. The lumen of the seminiferous tubules were full of spermatozoa similar to a sort of wave-like pattern. But SP animals showed primarily spermatogonia and some spermatocytes (SP, Comp Reg). The diameter of the tubules in SP animals was about less than half, which was equivalent to one eighth in volume compared to the LP animals.

In the animals treated with melatonin, the mass of testis of many animals was intermediate between active and inactive testis (Partial Reg). And the diameter of the seminiferous tubules of partially-regressed testis was in the midst of those of completely regressed testis and the nonregressed testis (Partial Reg). The thickness of the epithelial tissue was same as the testicular diameter. Spermatozoa and spermatids were observed in the lumen and the epithelium of the tubules of the testis. It was noticeable that some tubules showed germ cells in the lumen, which was speculated as the cells sloughed off the epithelia, implying that the degenerating process was underway.

The histological views of the epididymis were directly associated to those of testes examined above (Fig. 7). The spermatozoa were filled in epididymis of LP control animals (LP, Non-Reg) and were totally absent in the lumen of the epididymal tubules of SP control animals (SP, Comp Reg), which was consistent with the phenomena exhibited in the testis. In the testis regressed partially, spermatozoa were observed as the typical hooked shape of heads of the spermatozoa in the lumen (Partial Reg). Also, other round cells were witnessed, surmising as the germ cells and implying cells sloughed off the epithelium of the seminiferous tubule. That outcome might denote the degenerating process of the reproductive activities.

\section{DISCUSSION}

The administration of pure melatonin via gavage led to complete regression of testes in some animals treated with middle and high dosages of melatonin. These results indicate that the animals
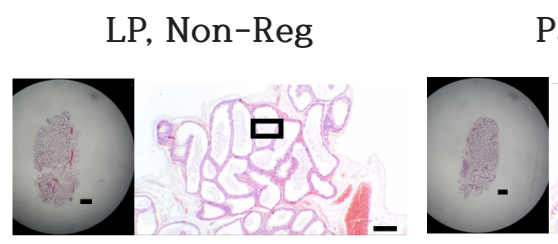

Partial Reg

SP, Comp Reg
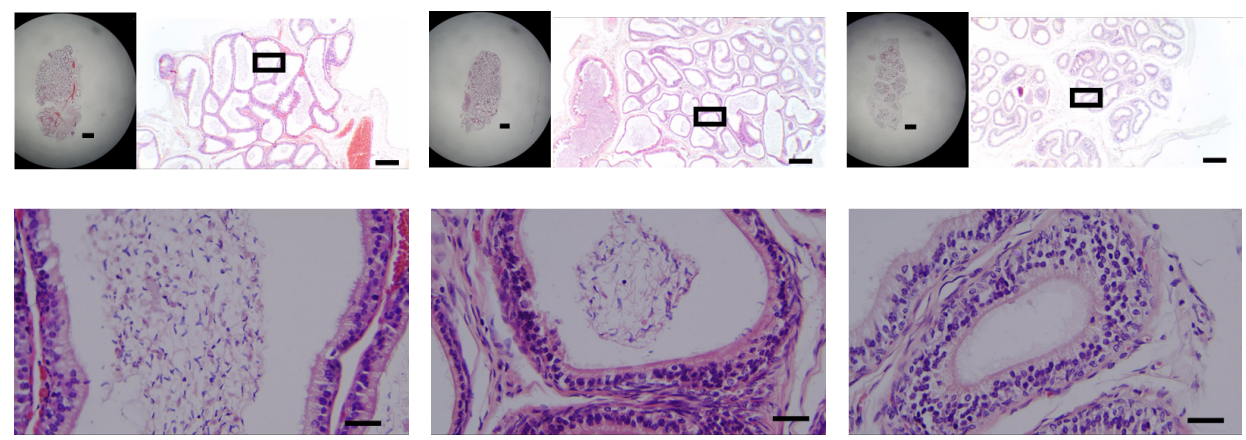

Fig. 7. Representative histological view of epididymis. The pictures at the left side in upper row show the entire shape of epididymis. LP, Non-Reg: epididymis of animals in long photoperiod (LP) control and animals who had non-regressed testis. Partial Reg: epididymis of animals who showed partial regression. SP, Comp Reg: epididymis of animals in short photoperiod (SP) control and animals who had completely regressed testis. The rectangles in the upper row are amplified in the lower row. Bar at the left picture in upper row $=1 \mathrm{~mm}$. Bar at the right picture in upper row $=50 \mu \mathrm{m}$. Bar in lower row=10 $\mu \mathrm{m}$ 
were sensitive enough to respond to the dosages of melatonin applied in this experiment. The effects of melatonin were supported by the histological examination of testis and epididymis, where no spermatozoa were observed. To our knowledge, these results are for the first time to show the inhibitory activity of the melatonin ingestion in blocking spermatogenesis in golden hamsters. And the incomplete regression of the reproductive activity of some animals is surmised that the degenerating process of the testes was underway.

The animals normally produce endogenous melatonin at night. The exogenous melatonin administered in the evening might combine to the endogenous melatonin, resulting in lengthened period of elevated level of melatonin as animals in winter season. Thus, it could be speculated that the extension of elevated melatonin suppressed the reproductive endocrine system, which is regulated by gonadotropin releasing hormone $(\mathrm{GnRH})$ neuronal cells in the hypothalamus. The secretion of $\mathrm{GnRH}$ is diminished and gonadotropins, follicle stimulating hormone and luteinizing hormone, accordingly are reduced, leading to degeneration of the testicular functions (Pickard \& Silverman, 1979).

In the previous report induced testicular regression, $15 \mu \mathrm{g}$ of melatonin per $\mathrm{kg}$ of body weight was subcutaneously injected daily into the nape of the neck (Stetson \& Tay, 1983). They found biphasic action of melatonin in which nearly 5 hours before lighting-out and 1 hour before lightson were effective to result in antifertility function of testes. The injections of the other times, such as morning, early afternoon, and night, of a day were ineffective to invoke sexual involution in the animals. Thus, the time of administration was set in the evening in the present investigation, which was 4 hours before the lights turned off.

The concentrations of melatonin used in this study was much higher than those reported previously (Stetson \& Tay, 1983). The reason that higher dose of melatonin was applied was that melatonin had to flow through the gastrointestinal tract. If melatonin is injected, all of melatonin due to hydrophobic nature could spread and react on all tissues of the whole body. If ingested, melatonin should pass through the digestive tract during which it could be decomposed into the ineffective component(s), absorbed into blood stream and exerted its effect, and excreted uselessly out of the body. Considering some of melatonin to be expended, higher amount of melatonin was subjected into the experimental animals. Nevertheless, the results were intriguing that melatonin ingestion induced largely same results as shown by melatonin injection.

Therefore, the action mechanism of melatonin could be speculated as follows. Melatonin swallowed by intubation into the body could primarily be easily absorbed, due to hydrophobic nature, in the gastrointestinal tract and then spread all over the body through the circulatory system (Choi \& Lee, 2012). As the action of gonadal steroid hormones, melatonin could reach and act on the hypothalamus, resulting in ultimately gonadal regression. It has been known that long term exposure to SP condition reduces the release of GnRH (Choi \& Han, 2010; Choi \& Lee, 2012). Also, in the similar manner, melatonin might act directly on the pituitary. Thus, melatonin results in functional involution of reproductive activity by reducing release of gonadotropins in this animal (Pickard \& Silverman 1979). Moreover, it can be speculated that melatonin affects directly the testes. It would operate on the Leydig cells to suppress the production of testosterone, and then the reduced amount of steroid influences the spermatogenesis.

The regressive role of melatonin on the reproductive activity of male golden hamsters is supported by the reduced testes as well as accompanied by the diminished accessory sex organs such as seminal vesicle and epididymis.

The present results additionally showed that melatonin also showed the incomplete blocking outcome in some animals, which might be due to the possible shortage of the period of treatment time. In case of golden hamsters, the period of 8 weeks has repeatedly been reported to be enough 
to testify the sexual regression. The possibility that longer administration could degenerate the sexual function in this animal is reasonably logical.

The resultant testes in this experiment could be classified into three categories by the different mass and the histological examination, which are active, inactive, and regression-undergoing testes. The active testes marked as non-reg, were large and showed the thickened epithelium of the seminiferous tubules containing full of germ cells, including spermatogonia, spermatocytes, and spermatids, and had abundant spermatozoa in the lumen. On the other hand, SP animals had thinned seminiferous tubules, showing the diameter of the tubules to be about less than half, which was equivalent to one eighth in volume compared to the testes of LP control animals. And SP animals showed primarily spermatogonia and some spermatocytes in the epithelial wall of the tubules. No spermatids and mature spermatozoa were observed at all in the epithelium and the lumen of the tubules.

In the animals treated with melatonin, the mass of testis of many animals was intermediate between active and inactive testis. The regression-undergoing testes marked as partial reg, had intermediate diameter of the seminiferous tubules between the completely regressed testis and the non-regressed testis. The thickness of the epithelial tissue was nearly same as the active testes. The energetic spermatogenesis full of germ cells were observed, including mature spermatozoa in the lumen. It was discernible that some tubules were absent of germ cells in the lumen, which was speculated as the cells sloughed off the epithelia, implying that the degenerating process was underway.

Likewise, the weights of the epididymides were directly parallel to the testes mentioned above. The epididymides of LP control animals and low dose melatonin dose group were filled with spermatozoa in the lumen of all tubules. The dark-stained and hooked heads of spermatozoa were evident and observed everywhere. SP control animals and animals possessed regressed testis showed no spermatozoa in the epididymides. In the testis regressed partially, spermatozoa were observed as the typical hooked shape of heads of the spermatozoa in the lumen. Also, some of other round cells were witnessed, surmising as the germ cells and implying cells sloughed from the epithelium of the seminiferous tubule. The consequence might denote the degenerating process of the reproductive activities.

In conclusion, the present results imply that the ingestion of pure melatonin itself affects the reproductive system and causes the testis to regress. Accordingly, these outcomes suggest that phytomelatonin in the feed could interfere the reproductive function. Further investigation is required to find whether dietary melatonin could alter the reproductive function.

\section{REFERENCES}

Arnao MB, Hernández-Ruiz J (2018) The potential of phytomelatonin as a nutraceutical. Molecules 23:238-256.

Bonomini F, Borsani E, Favero G, Rodella LF, Rezzani R (2018) Dietary melatonin supplementation could be a promising preventing/therapeutic approach for a variety of liver diseases. Nutrients 10:1135-1159.

Choi D (2001) Influence of melatonin on reproductive function in male golden hamsters. Dev Reprod 5:1-8.

Choi D (2013a) Potency of melatonin in living beings. Dev Reprod 17:149-177.

Choi D (2013b) Continuous melatonin attenuates the regressing activities of short photoperiod in male golden hamsters. Dev Reprod 17:111-119. 
Choi D (2019) Effects of dietary supplement containing melatonin on reproductive activity in male golden hamsters. Dev Reprod 23:101-110.

Choi D, Han E (2010) The impacts of photoperiods on hypothalamic proteins in the reproductive activities of golden hamsters. Dev Reprod 14:185-197.

Choi D, Roh HS, Kang DW, Lee JS (2014) The potential regressive role of Syzygium aromaticum on the reproduction of male golden hamsters. Dev Reprod 18:57-64.

Choi D, Lee SH (2012) Neuroendocrine system in seasonal breeder: focusing on the reproductive activity of male golden hamster. Dev Reprod 16:1-8.

Dubbels R, Reiter RJ, Klenke E, Goebel A, Schnakenberg E, Ehlers C, Schiwara HW, Schloot W (1995) Melatonin in edible plants identified by radioimmunoassay and by high performance liquid chromatography-mass spectrometry.J Pineal Res 18:28-31.

Elliott JA (1976) Circadian rhythms and photoperiodic time measurement in mammals. Fed Proc 35:2339-2346.

Gaston S, Menaker M (1967) Photoperiodic control of hamster testis. Science 158:925-928.

Grosse J, Maywood ES, Ebling FJP, Hastings MH (1993) Testicular regression in pinealectomized Syrian hamsters following infusions of melatonin delivered on non-circadian schedules. Biol Reprod 49:666-674.

Hattori A, Migitaka H, Iigo M, Itoh M, Yamamoto K, Ohtani-Kaneko R, Hara M, Suzuki T, Reiter RJ (1995) Identification of melatonin in plants and its effects on plasma melatonin levels and binding to melatonin receptors in vertebrates. Biochem Mol Biol Int 35:627-634.

Hiebert SM, Green SA, Yellon SM (2006) Daily timed melatonin feedings mimic effects of short days on testis regression and cortisol in circulation in Siberian hamsters. Gen Comp Endocrinol 146:211-216.

Lee JS, Oh HA, Kwon JY, Jeong MH, Lee JS, Kang DW, Choi D (2013) The effects of Cynomorium songaricum on the reproductive activity in male golden hamsters. Dev Reprod 17:37-43.

Lerner AB, Case JD, Takahashi Y, Lee TH, Mori W (1958) Isolation of melatonin, the pineal gland factor that lightens melanocytes.J Am Chem Soc 80:2587.

Maywood ES, Lindsay JO, Karp J, Power JB, Williams LM, Titchener L, Ebling FJP, Herbert J, Hasting MH (1991) Occlusion of the melatonin-free interval blocks the short day gonadal response of the male Syrian hamster to programmed melatonin infusions of necessary duration and amplitude. J Neuroendocrinol 3:331-337.

Meng X, Li Y, Li S, Zhou Y, Gan RY, Xu DP, Li HB (2017) Dietary sources and bioactivities of melatonin. Nutrients 9:367-430.

Oladi E, Mohamadi M, Shamspur T, Mostafavi A (2014) Spectrofluorimetric determination of melatonin in kernels of four different Pistacia varieties after ultrasound-assisted solid-liquid extraction. Spectrochim Acta A Mol Biomol Spectrosc 132:326-329.

Paredes SD, Korkmaz A, Manchester LC, Tan DX, Reiter RJ (2009) Phytomelatonin: A review. J Exp Bot 60:57-69.

Pickard GE, Silverman AJ (1979) Effects of photoperiod on hypothalamic luteinizing hormone releasing hormone in the male hamster. J Endocrinol 83:421-428.

Ramakrishna A, Giridhar P, Sankar KU, Ravishankar GA (2012) Melatonin and serotonin profiles in beans of coffee species.J Pineal Res 52:470-476.

Reiter RJ (1980) The pineal and its hormones in the control of reproduction in mammals. Endocr Rev 1:109-131.

Rollag MD, Panke ES, Trakulrungsi W, Trakulrungsi C, Reiter RJ (1980) Quantification of daily melatonin synthesis in the hamster pineal gland. Endocrinology 106:231-236. 
Stetson MH, Rollag MD, Watson-Whitmyre M, Tate-Ostroff B (1983) The effect of daily injections and constant release implants of melatonin on the endogenous pineal melatonin rhythm in golden hamsters. Proc Soc Exp Biol Med 174:119-122.

Stetson MH, Tay DE (1983) Time course of sensitivity of golden hamsters to melatonin injections throughout the day. Biol Reprod 29:432-438.

Stetson MH, Watson-Whitmyre M (1984) Physiology of the pineal and its hormone melatonin in annual reproduction in rodents. In: Reiter RJ (ed), The Pineal Gland. Raven Press, New York, NY, pp 109-153.

Stetson MH, Watson-Whitmyre M (1986) Effects of exogenous and endogenous melatonin on gonadal function in hamsters. J Neural Transm 21:55-80.

Sugden D (1989) Melatonin biosynthesis in the mammalian pineal gland. Experientia 45:922-932.

Watson-Whitmyre M, Stetson MH (1985) A mathematical method for estimating paired testes weight from in situ testicular measurements in three species of hamster. Anat Rec 213:473-476. 
